Supplement of Nat. Hazards Earth Syst. Sci., 20, 3439-3454, 2020

https://doi.org/10.5194/nhess-20-3439-2020-supplement

(c) Author(s) 2020. This work is distributed under

the Creative Commons Attribution 4.0 License.

(c) (1)

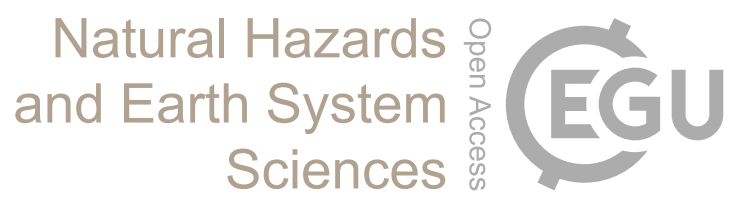

Supplement of

\title{
A classification scheme to determine wildfires from the satellite record in the cool grasslands of southern Canada: considerations for fire occurrence modelling and warning criteria
}

Dan K. Thompson and Kimberly Morrison

Correspondence to: Dan K. Thompson (Daniel.Thompson@canada.ca)

The copyright of individual parts of the supplement might differ from the CC BY 4.0 License. 


\section{Supplementary Material}

\section{S1 Methods}

Supplementary methods were included to provide detailed methodology beyond what was practical in the main article.

\section{S1.1 Creation of MODIS hotspot table with required attributes}

5 We took the filtered MODIS hotspots (MOD14A1 and MYD14A1) (Giglio, 2015), found within our study area, and ensured there were no missing values within required attributes (such as Fire Weather Index values, date, or time). This data table contained weather, Fire Weather Index (FWI) System values, grass percent cured and Fire Radiative Power (FRP) values for each hotspot. UTC dates and times were converted to local time and Julian Date (day of year). Since the MODIS satellites have both a daytime and nighttime overpass, these times were used to classify the hotspots into day and night observations

10 using the following rules:

1. If the rounded hotspot hour was observed from 800 to 1700 LST, the hotspot was considered a 'Day' observation.

2. If the rounded hotspot hour was observed from 1700 to $800 \mathrm{LST}$, the hotspot was considered a 'Night' observation.

3. If the hotspot was part of the night observation, but after midnight, the Julian Date was modified to match the before midnight observations (one day was subtracted).

\section{$15 \quad$ S1.2 Creation of hotspot clusters}

Since any given fire can result in one to many hotspot detections it was decided to group hotspots by date, over-pass time (day versus night) and location in an attempt to group hotspots that represent the same fire together. These groupings are called hotspot clusters in this study. Hotspots are considered to be part of the same cluster if they were from the same date, part of the same overpass time and within a certain distance of each other. This distance was determined based on the track and scan length of the MODIS satellite for each hotspot. At nadir the track and scan length of the MODIS satellites is $1 \mathrm{~km}$, giving a pixel resolution of $1 \mathrm{~km}$ by $1 \mathrm{~km}$. The farther away from nadir the observation, the larger the track and/or scan length will be. Therefore hotspots will be spaced farther apart the farther from nadir they were observed. This also means the hotspot could be located anywhere within the given track and scan distance. To compensate for this each hotspot was given a circular buffer based on its track and scan distance, using the following procedure.

The area of an ellipse was calculated using the track and scan distances as the two diameters (Eq. (S1)):

$A_{\mathrm{ELLIPSE}}=\pi *\left(\frac{D_{\mathrm{SCAN}}+D_{\mathrm{TRACK}}}{4}\right)^{2}$,

where $D_{\text {SCAN }}$ is the scan distance of the MODIS hotspot and $D_{\text {TRACK }}$ is the track distance of the MODIS hotspot. 
The radius of a circle that has the same area as the above ellipse ( $\left.A_{\text {ELLIPSE }}\right)$ was calculated. This radius was multiplied by 1.05 to increase overlap between hotspots belonging to the same fire (Eq. (S2)):

$R_{\mathrm{BUF}}=\sqrt{A_{\mathrm{ELLIPSE}} / \pi} \cdot 1.05$.

The idea was to have a buffered area the same as the pixel size of the MODIS scan for any given hotspot. This buffer also give us the extent of the area that a fire from a hotspot detection could actually be located and from this calculate the fuel type it could be burning in. It also allowed us to merge the hotspots that were likely part of the same fire into hotspot clusters. The hotspot clusters had their attributes, such as weather, merged as well. An attribute was merged by max value or mean, for each

40 hotspot cluster. FFMC, DMC, DC, ISI, BUI, FWI, precipitation, relative humidity, wind speed, temperature, hour of detection, percent cured and NDVI were merged by mean. HFI and FRP were merged by max values.. After observing these hotspot clusters it was noted that not all hotspots that were known to be part of the same fire were merging. This is likely a result of averaging the hotspot's track and scan distance when there was a large difference between the two. Five percent was added to the buffer radius to fix this.

\section{S1.3 Classifying grass fires versus forest fires}

The percent grass cover for each hotspot cluster was then determined. The number of pixel centers from each AAFC Land Use type (Agriculture and Agri-Food Canada, 2018) that fell within a hotspot cluster were counted. From this the percent grass was calculated by dividing the number of grass pixels by the number of fuel pixels. Grass pixels include: 'managed grasslands', 'unmanaged grasslands' and 'cropland'. Additionally, if there were 5\% or more grass in a given hotspot cluster

50 using the fore mentioned grass pixels, 'undifferentiated wetlands' were included in the grass pixel count. All pixels except 'settlement', 'roads', ‘water', 'other land' or 'unclassified' are considered fuel.

The land use raster had a vegetation classification of 'undifferentiated wetlands' that appeared to cover a wide variety of vegetation types. When it was close to agriculture and other grasslands, it was often grassland-like vegetation (e.g. cattails in

55 sloughs or pothole wetlands). In the more forested areas these 'undifferentiated wetlands' seemed to be more like bogs and fens. It was decided that 'undifferentiated wetlands' could sometimes be considered as a potential fire spread pathway for the purposes of this study. If the hotspot cluster had $5 \%$ or more grass (cropland, 'unmanaged grassland' and 'managed grassland') then the 'undifferentiated wetlands' were included as grass, otherwise they were included as fuel, non-grass. 


\section{S1.4 Known wildfire and agricultural fire hotspot database creation}

60 A model to classify grass hotspot clusters as either belonging to a wildfire or an agricultural fire was created. In order to train this model, a database of known wildfire and agricultural fire hotspot clusters was created. The Canadian National Fire Database (CNFDB) (Hanes et al., 2018) and evacuation records were used to identify hotspot clusters which are known to be wildfires. CNFDB fires which are not wildfires are listed as prescribed burns within the CNFDB. The CNFDB data is from the official fire agency records and collated nationally by the Canadian Forest Service. The processed Landsat 8 imagery

65 (Kato et al., 2018), and to a small extent the CNFDB, were used to identify hotspot clusters that were considered agricultural fires.

The CNFDB contains polygons of known wildland fires within Canada. The record is incomplete, especially within the grassland area. However, if a fire is recorded, it is a known wildland fire and unless otherwise indicated, a wildfire. The following criteria were used to determine which hotspot clusters matched CNFDB polygons:

It was considered to be a match between hotspot cluster(s) and a CNFDB polygon if:

1. If the hotspot cluster overlaps the CNFDB polygon (Fig. S1) and the hotspot cluster date is no later than 8 days after or 3 days before the recorded start date for CNFDB grass fires. OR;

2. If there is no fire report date (usually start date), but there is a polygon date for the CNFDB polygon:

a. The hotspot clusters and CNFDB polygon overlap significantly, were from the same year, and the polygon date was not from before the hotspot cluster date. OR;

3. If there is no fire report date (usually start date) and no polygon date for the CNFDB polygon:

a. The hotspot clusters and CNFDB polygon overlap significantly and were from the same year. It is assumed the same patch will not burn twice in the same year.

Evacuation records (largely derived from media reports) were used to indicate when and where there were wildfires. An evacuation, due to direct threat from fire, indicates that not only is there a wildfire near the community, but that the conditions are such that any fire in the immediate area is almost certainly also a wildfire. The evacuation database might not have captured the exact same fire as a hotspot cluster, but we deemed any hotspot cluster within $50 \mathrm{~km}$ of an evacuation to be similarly threatening. Multiple grass fire ignitions are common on days of extreme fire danger. Since wind conditions in relatively flat, grassy areas, should be relatively constant, a $50 \mathrm{~km}$ radius around the evacuation point was considered to have similar weather conditions as the evacuation point. The following criteria were used to determine which hotspot clusters should be included in the database as wildfires based on the evacuation databases. The hotspot cluster was included as a wildfire if:

1. The evacuation point and hotspot cluster are within the study area. AND;

2. The hotspot cluster is within a $50 \mathrm{~km}$ radius of an evacuation. AND;

3. The evacuation is from a direct threat (If it was caused by smoke or if it was unknown, it was not used). AND; 
4. The hotspot cluster is from within the first day of said evacuation.

Finally, processed Landsat 8 fire detections and image subset were used to identify agricultural fires. This method was only 95 used to identify agricultural field, pile and, windrow burns as these fires often have regular and identifiable shaped burns (Fig. 3). The fires identified in the Landsat imagery were considered an agricultural fire if:

1. The fire and burn scar were contained within one field or within more than one field but clearly intentionally. This means the burn scar/flames should follow the rectangular shape of the field/fields being burned. In either case there should be no odd shaped excursions. AND;

2. The fire should not be elliptical in shape. Since most agricultural fires are started from a line ignition, and most wildfires are started by a point ignition an elliptical shape could indicate a wildfire. If it was ambiguous, the fire was not included in the database. AND;

3. The fire should have flame only or flame and a burn scar to ensure field was being burned and not plowed.

105 Provided the above three conditions were met, the following specific situations were also considered agricultural fires:

1. A continuous line of thin flame which boarded one or more sides of a field(s). OR;

2. The burning occurred in rows (windrow burning or a controlled ignition technique) or semi-regular dots (pile burning).

Once an agricultural fire was identified from the Landsat imagery, hotspot clusters were included in the database as an 110 agricultural fire if:

1. They were from the same date as the Landsat imagery. AND;

2. They at least partially overlapped with the field being burned. The part of the field that overlapped with the hotspot cluster did not have to be the part being actively burned in the Landsat imagery, so long as it looked like the intent was to burn the whole field.

115 This is not a complete record of agricultural fire observed by the processed Landsat imagery from 2013-2018. There were many fires that could not be confidently classified based on shape. Additionally, a thorough search was not done to ensure all classifiable fires within the timeframe specified were found. Database creation stopped once there were enough examples to build the model. The final database contained 41 hotspot clusters (104 hotspots) that represent 40 'known' examples of agricultural fires and 99 hotspot clusters (386 hotspots) that are 'known' examples of wildfires. This database was used to create a model which was then used to classify all 24297 hotspot clusters as wildfires or agricultural fires.

\section{References}

Agriculture and Agri-Food Canada: Land Use 2010, [online] Available from: https://open.canada.ca/data/en/dataset/9e1efe92e5a3-4f70-b313-68fb1283eadf (Accessed 9 March 2020), 2018. 
Giglio, L.: MODIS Collection 6 Active Fire Product User's Guide Revision A, Department of Geographical Sciences, 125 University of Maryland. [online] Available from: https://lpdaac.usgs.gov/documents/88/MOD14_User_Guide_v6.pdf, 2015.

Hanes, C. C., Wang, X., Jain, P., Parisien, M.-A., Little, J. M. and Flannigan, M. D.: Fire-regime changes in Canada over the last half century, Can. J. For. Res., 49(3), 256-269, doi:10.1139/cjfr-2018-0293, 2018.

Kato, S., Kouyama, T., Nakamura, R., Matsunaga, T. and Fukuhara, T.: Simultaneous retrieval of temperature and area 130 according to sub-pixel hotspots from nighttime Landsat 8 OLI data, Remote Sensing of Environment, 204, 276-286, doi:10.1016/j.rse.2017.10.025, 2018. 


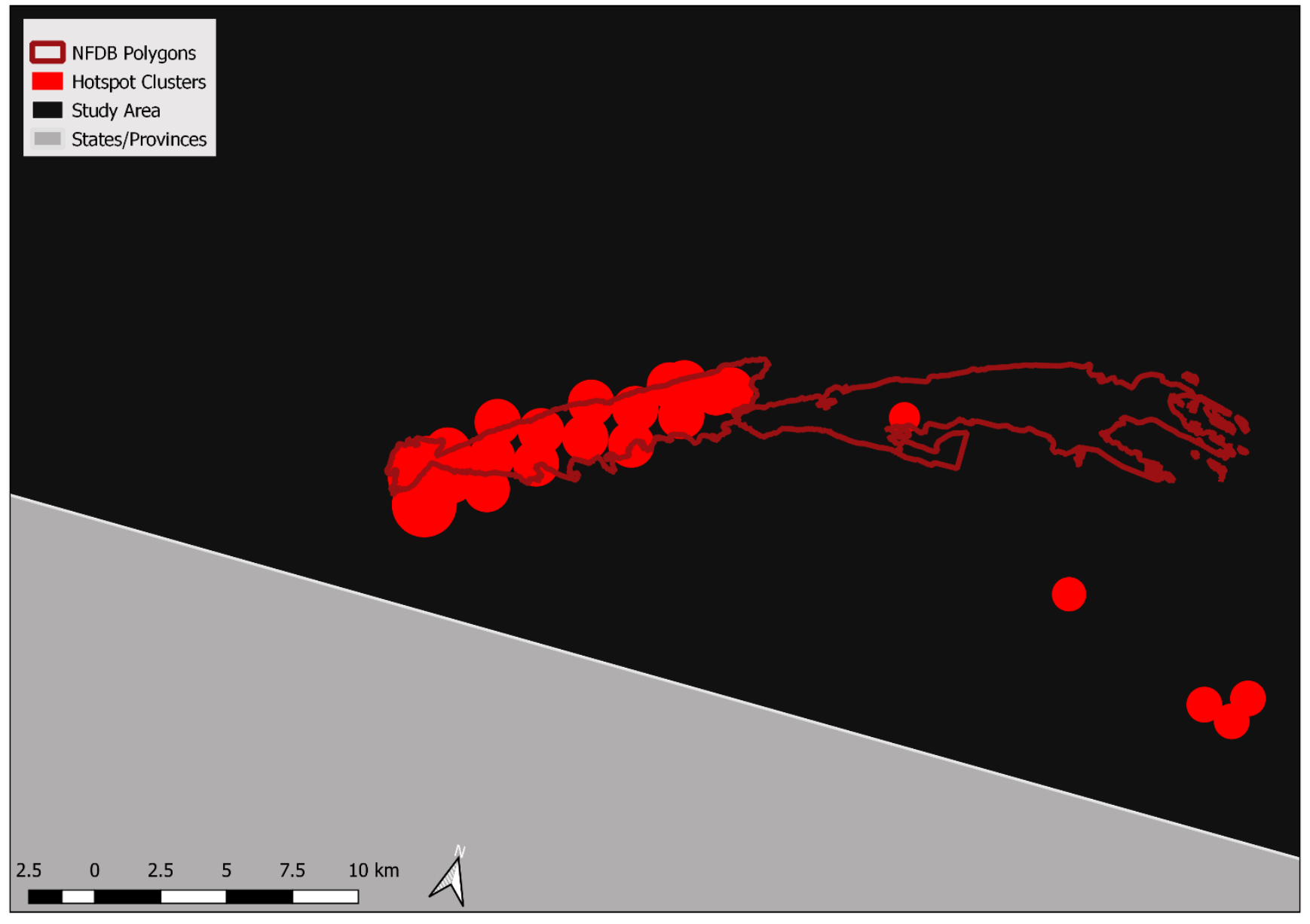

135 Figure S1: Example of hotspot clusters that overlap with a CNFDB polygon. 
Figure S2: Appendix A. Observation density biplots of the fire weather associated with thermal detections $(n=1700)$ where the Initial Spread Index of the Canadian Fire Weather Index System is 17 or higher (1,700 of 24,316 hotspot detection clusters).

140 Panel A: noon vapour pressure deficit vs wind speed (both local noon standard time) for all observations of ISI 17 or higher. Panel B: relative humidity vs wind speed for the same subset. Panel C: Fine Fuel Moisture Code vs wind speed. 

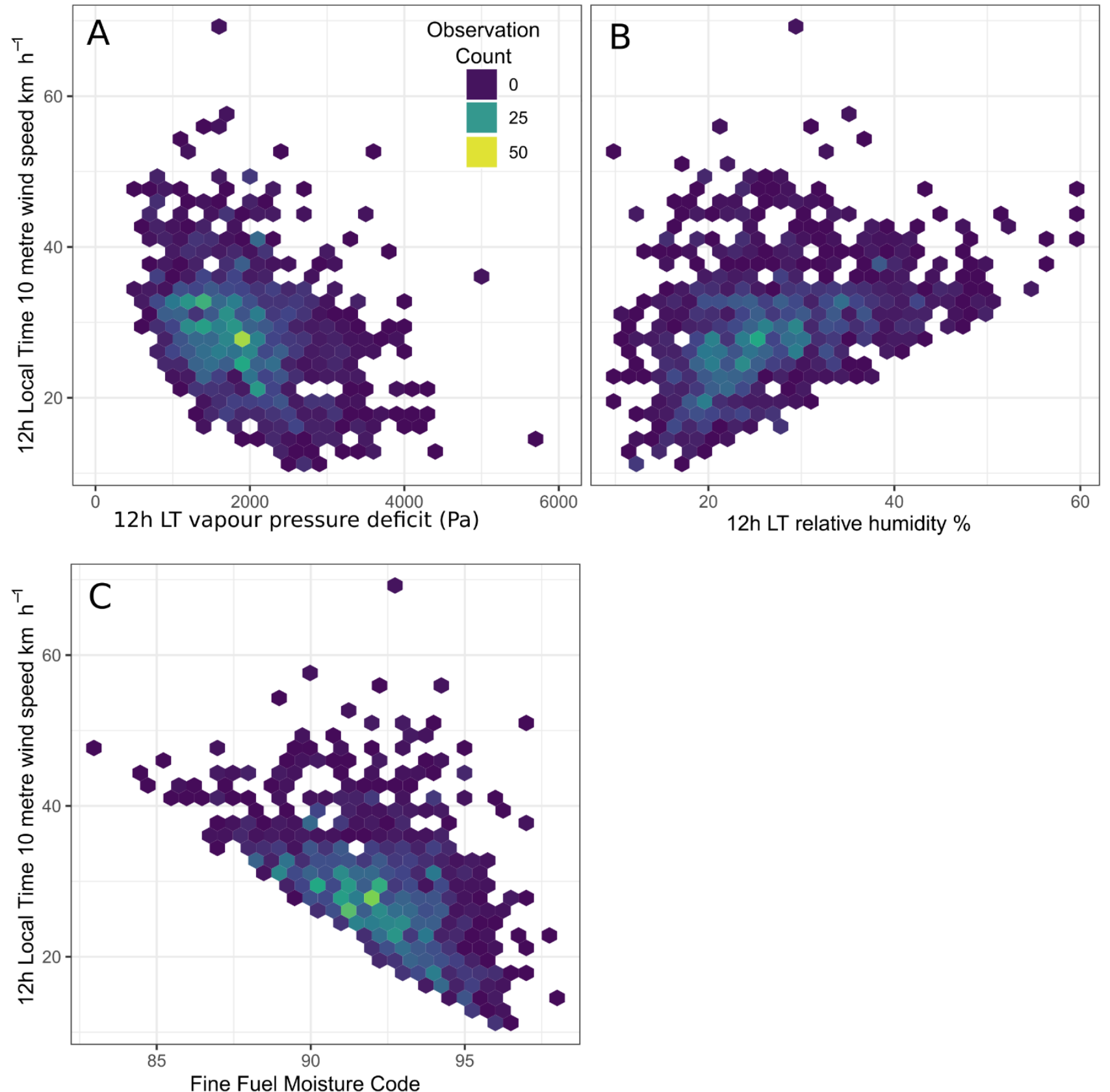

Fine Fuel Moisture Code 